\title{
Nonexistence results for Hadamard-like matrices
}

\author{
Justin D. Christian and Bryan L. Shader \\ Department of Mathematics, University of Wyoming, USA \\ christianjd@yahoo.com, bshader@uwyo.edu
}

Submitted: Aug 26, 2003; Accepted: Jan 19, 2004; Published: Jan 23, 2004

MR Subject Classifications: 05B20,15A36

\begin{abstract}
The class of square $(0,1,-1)$-matrices whose rows are nonzero and mutually orthogonal is studied. This class generalizes the classes of Hadamard and Weighing matrices. We prove that if there exists an $n$ by $n(0,1,-1)$-matrix whose rows are nonzero, mutually orthogonal and whose first row has no zeros, then $n$ is not of the form $p^{k}, 2 p^{k}$ or $3 p$ where $p$ is an odd prime, and $k$ is a positive integer.
\end{abstract}

\section{Introduction}

A Hadamard matrix of order $n$ is an $n$ by $n(1,-1)$-matrix $H$ satisfying $H H^{T}=n I$, where $I$ denotes the identity matrix and $H^{T}$ denotes the transpose of $H$. Hadamard matrices were first introduced by J. Hadamard in 1893 as solutions to a problem about determinants (see [GS, WSW]). The following well-known, simple result shows that the standard necessary condition (that is, $n=1, n=2$, or $n \equiv 0 \bmod 4$ ) for the existence of a Hadamard matrix of order $n$, is a consequence of the mutual orthogonality of three $(1,-1)$-vectors.

Proposition 1 Let $u, v$, and $w$ be mutually orthogonal, 1 by $n(1,-1)$-vectors. Then $n \equiv 0 \bmod 4$.

Proof. Each entry in the vectors $u+v$ and $u+w$ is even. Hence $(u+v) \cdot(u+w)$ is a multiple of 4 . Since $(u+v) \cdot(u+w)=u \cdot u=n$, the result follows.

The famous Hadamard Conjecture asserts that there exists a Hadamard matrix of order $n$ for every $n \equiv 0 \bmod 4$, and has been verified for $n<428$ (see [HKS]).

Weighing matrices are generalizations of Hadamard matrices. Let $n$ and $w$ be positive integers. An $(n, w)$-weighing matrix is an $n$ by $n(0,1,-1)$-matrix $W=\left[w_{i j}\right]$ satisfying $W W^{T}=w I$. Weighing matrices have been extensively studied (see $[\mathrm{C}]$ and the references therein). Several necessary conditions for the existence of an $(n, w)$-weighing matrix are known. If $n>1$ is odd, then necessarily $w$ is a perfect square and $n \geq w+\sqrt{w}+1$ with 
equality only if there exists a projective plane of order $\sqrt{w}$. The first of these conditions follows from taking determinants of both sides of $W W^{T}$, and the second from bounding the number of nonzero entries of $C C^{T}$, where $C=\left[c_{i j}\right]$ is the $n$ by $n$ matrix with $c_{i j}=1$ when $w_{i j}=0$, and $c_{i j}=0$ when $w_{i j} \neq 0$. If $n \equiv 2 \bmod 4$, then necessarily $w$ is a sum of two squares, and $n \leq 2$ or $w<n$. The first of these conditions is a number theoretic consequence of applying Witt's cancellation to the congruence $W W^{T}=w I$, and the latter follows from the standard necessary condition for Hadamard matrices. The Weighing Matrix Conjecture asserts that there exists an $(n, w)$-weighing matrix for each $n$ of the form $n \equiv 0 \bmod 4$ and each $w \leq n$. This conjecture has been confirmed for $n \leq 88$.

In this note we consider related combinatorial problems. A matrix $A$ is row-orthogonal if each of its rows is nonzero, and its rows are mutually orthogonal. Thus the $(0,1,-1)$ row-orthogonal matrices generalize both Hadamard and Weighing matrices. The sparsity of row-orthogonal $(0,1,-1)$-matrices (actually their transposes) has been studied in [GZ]. A row or column of $A$ is full if each of its entries is nonzero. Each row of a Hadamard matrix is full, and a weighing matrix has a full row if and only if it is a Hadamard matrix. The problem studied in this note is: determine the positive integers $n$ for which there exists an $n$ by $n$, row-orthogonal $(0,1,-1)$-matrix with a full row. As such matrices are not required to have the same type of regularity (i.e. each row and column has the same number of nonzeros) as Hadamard and Weighing matrices, it appears that suitably adapted, and even different techniques are needed to study this problem.

In section 2, we develop some basic properties of row-orthogonal $(0,1,-1)$-matrices. In section 3, we use these basic properties to give several non-existence results. We show that the existence of a row-orthogonal $(0,1,-1)$-matrix of order $n$ with full column is equivalent to the existence of a Hadamard matrix of order $n$. We also show that a roworthogonal $(0,1,-1)$-matrix of order $n$ with a full row does not exist if $n$ has the form $p^{k}$, $2 p^{k}$, or $3 p$ where $p$ is an odd prime and $k$ is a positive integer.

\section{Basic Results}

In this section we establish some notation and observe several basic results that we will use throughout the remainder of the note. We are interested in determining the $n$ for which there exists a row-orthogonal $(0,1,-1)$-matrix $A$ of order $n$ with a full row. Note that scaling certain rows of a row-orthogonal $(0,1,-1)$-matrix results in a row-orthogonal $(0,1,-1)$-matrix. Certainly if $H$ is a Hadamard matrix, then $H$ satisfies our requirements of row orthogonality and full row. Thus, if the Hadamard conjecture is true, then such an $A$ exists for $n=1, n=2$, and $n=4 k$ for each positive integer $k$. Two questions, which we only begin to study here, come to mind: can one prove the existence of such $A$ of order $n=4 k$ for each positive integer $k$ ? and must such a matrix have order 1 , 2, or $4 k$ for some positive integer $k$ ?

Each of the matrices below is an example of a square, row-orthogonal $(0,1,-1)$-matrix 
with a full row.

$$
[1], \quad\left[\begin{array}{rr}
1 & 1 \\
1 & -1
\end{array}\right], \quad\left[\begin{array}{rrrr}
1 & 1 & 1 & 1 \\
1 & 1 & -1 & -1 \\
1 & -1 & 0 & 0 \\
0 & 0 & 1 & -1
\end{array}\right]
$$

The number of nonzero entries in row $i$ of $A$ will be denoted by $e_{i}$. Note that if $A$ is a row-orthogonal $(0,1,-1)$-matrix, then $A A^{T}$ is a diagonal matrix $D$ whose $j$ th diagonal entry is $e_{j}$. A simple consequence is the following:

Proposition 2 Let $A$ be an $n$ by $n$ row-orthogonal $(0,1,-1)$-matrix. Then $e_{1} e_{2} \cdots e_{n}$ is a perfect square.

Proof. This follows immediately from $e_{1} e_{2} \cdots e_{n}=\operatorname{det}\left(A A^{T}\right)=(\operatorname{det} A)^{2}$.

Let $A=\left[a_{i j}\right]$ be an $n$ by $n$ row-orthogonal matrix. For $j=1,2, \ldots, n$, let $\alpha_{j}=\{i$ : $\left.a_{i j} \neq 0\right\}$. Since no row of $A$ is the zero row, each $e_{j}$ is positive, and we define $Q$ by

$$
Q=\operatorname{diag}\left(\frac{1}{\sqrt{e_{1}}}, \frac{1}{\sqrt{e_{2}}}, \ldots, \frac{1}{\sqrt{e_{n}}}\right) A .
$$

Since $Q$ is the matrix obtained from $A$ by normalizing each of its rows to have length $1, Q$ is an orthogonal matrix. In particular, the columns of $Q$ have length 1, and are mutually orthogonal. This implies the following:

Proposition 3 Let $A$ be an $n$ by $n$, row-orthogonal matrix. Then

$$
\begin{aligned}
\sum_{i \in \alpha_{j}} \frac{1}{e_{i}} & =1, \quad \text { for } 1 \leq j \leq n, \text { and } \\
\sum_{i \in \alpha_{j} \cap \alpha_{k}} \frac{a_{i j} a_{i k}}{e_{i}} & =0, \quad \text { for } 1 \leq j<k \leq n .
\end{aligned}
$$

Proposition 3 indicates that the study of row-orthogonal $(0,1,-1)$-matrices will involve sums of reciprocals of integers. The following proposition concerns such sums. Let $p$ be a prime and let $Q_{p}$ denote all rationals $q$ such that $q$ can be expressed as the ratio of integers $\frac{r}{s}$, where $p$ does not divide $s$. It is well known that $Q_{p}$ (with the usual addition and multiplication) is a ring. The following is an immediate consequence of the fact that $Q_{p}$ is closed under addition and subtraction.

Proposition 4 Let $f_{1}, f_{2}, \ldots, f_{n}, g_{1}, g_{2}, \ldots, g_{n}$ be integers and let $p$ be a prime such that $\sum_{j=1}^{n} \frac{g_{j}}{f_{j}}$ is an integer, $f_{j} \equiv 0 \bmod p$ for $j=1,2, \ldots, k$, and $f_{j} \not \equiv 0 \bmod p$ for $j=$ $k+1, \ldots, n$. Then $\sum_{j=1}^{k} \frac{g_{j}}{f_{j}} \in Q_{p}$. 


\section{Non-existence Results}

We will begin by considering row-orthogonal matrices with a full column.

Theorem 5 Let $A$ be a row-orthogonal $n$ by $n(0,1,-1)$-matrix with a full column. Then $A$ is a Hadamard matrix.

Proof. Since $A$ has a full column and each $e_{i} \leq n$, Proposition 3 implies that

$$
1=\frac{1}{e_{1}}+\frac{1}{e_{2}}+\cdots+\frac{1}{e_{n}} \geq \frac{1}{n}+\frac{1}{n}+\cdots+\frac{1}{n}=1
$$

Thus $e_{i}=n$ for each $i$. Therefore $A$ is Hadamard.

Next we study square, row-orthogonal matrices with a full row. Interestingly, the condition of full row has much different consequences than the condition of full column. For example, the matrix

$$
A=\left[\begin{array}{rrrr}
1 & 1 & 1 & 1 \\
1 & 1 & -1 & -1 \\
1 & -1 & 0 & 0 \\
0 & 0 & 1 & -1
\end{array}\right]
$$

is row-orthogonal with a full row, but is not a Hadamard matrix. So for which $n$ does an $n$ by $n$ row-orthogonal matrix with a full row exist? We have already seen examples for $n=1, n=2$, and $n=4$. And, to date, we know of no row-orthogonal $(0,1,-1)$-matrix with a full row whose order is not the order of a Hadamard matrix. Each of the results in the remainder of the note indicate that there are some severe restrictions on the possible order of a row-orthogonal $(0,1,-1)$-matrix with a full row.

Theorem 6 Let $A$ be an $n$ by $n$ row-orthogonal $(0,1,-1)$-matrix with a full row. Then $n$ is not an odd prime.

Proof. Suppose to the contrary that $n$ is an odd prime. Since $n$ is odd, no two full 1 by $n(0,1,-1)$-vectors are orthogonal. By Proposition 2 , and the fact that $n$ is prime, $A$ has an even number of full rows. Therefore, $A$ has no full rows, contrary to assumption.

The 7 by 7 row-orthogonal matrix

$$
A=\left[\begin{array}{rrrrrrr}
0 & 0 & 1 & 0 & 1 & 1 & -1 \\
-1 & 0 & 0 & 1 & 0 & 1 & 1 \\
1 & -1 & 0 & 0 & 1 & 0 & 1 \\
1 & 1 & -1 & 0 & 0 & 1 & 0 \\
0 & 1 & 1 & -1 & 0 & 0 & 1 \\
1 & 0 & 1 & 1 & -1 & 0 & 0 \\
-1 & 1 & 0 & 1 & 1 & -1 & 0
\end{array}\right]
$$

shows the necessity of the assumption that $A$ has a full row in Theorem 6 . We now generalize the previous result to include all powers of an odd prime. 
Theorem 7 Let $A$ be an $n$ by $n$ row-orthogonal $(0,1,-1)$-matrix with a full row. Then $n$ is not of the form $p^{k}$ where $p$ is an odd prime $p$ and $k$ is a positive integer.

Proof. Suppose to the contrary that $n=p^{k}$ for some odd prime $p$ and positive integer $k$. Without loss of generality we can take the first row of $A$ to be full. By Proposition 3, we have

$$
\frac{1}{p^{k}}+\sum_{i \in \alpha_{1} \backslash\{1\}} \frac{1}{e_{i}}=1 .
$$

Multiplying by $p^{k-1}$ gives the equation

$$
\frac{1}{p}+\sum_{i \in \alpha_{1} \backslash\{1\}} \frac{p^{k-1}}{e_{i}}=p^{k-1} .
$$

Proposition 4 implies that at least one of the fractions $\frac{p^{k-1}}{e_{i}}$ is not in $Q_{p}$. Hence, $p^{k}$ divides $e_{i}$ for some $i \neq 1$. It follows that $A$ has two full rows, which is a contradiction-two $n$ by $1(1,-1)$-vectors are not orthogonal when $n$ is odd.

We next show nonexistence for $n$ of the form $n=2 p^{k}, p$ and odd prime.

Theorem 8 Let $A$ be a row-orthogonal $(0,1,-1)$-matrix with a full row. Then $n$ is not of the form $2 p^{k}$ where $p$ is an odd prime and $k$ is a positive integer.

Proof. Suppose to the contrary that $n=2 p^{k}$ for some odd prime $p$ and positive integer $k$. Suppose $A$ has $f$ full rows, and without loss of generality that these are the first $f$ rows of $A$. Consider column 1 of $A$. By Proposition 3, we have

$$
\frac{f}{2 p^{k}}+\sum_{i \in \alpha_{1} \backslash\{1,2, \ldots, f\}} \frac{1}{e_{i}}=1 .
$$

Multiplying by $p^{k-1}$ gives the equation

$$
\frac{f}{2 p}+\sum_{i \in \alpha_{1} \backslash\{1,2, \ldots, f\}} \frac{p^{k-1}}{e_{i}}=p^{k-1} .
$$

Suppose that $p$ does not divide $f$. By Proposition 4, there is an $i \in \alpha_{i} \backslash\{1, \ldots, f\}$ such that $p^{k}$ divides $e_{i}$. Since the first and $i$ th row of $A$ are orthogonal, $e_{i}$ is even. Hence, $n \geq e_{i} \geq 2 p^{k}$. This implies that row $i$ is full, contrary to assumption.

Thus, $p$ divides $f$. This implies that $A$ has at least 3 full rows, and Proposition 1 further implies that $n \equiv 0 \bmod 4$ - a contradiction.

We conclude this note, by proving nonexistence for $n$ of the form $3 p, p$ an odd prime.

Theorem 9 Let $A$ be a row-orthogonal $(0,1,-1)$-matrix with a full row. Then $n$ is not $3 p$ for some odd prime $p$. 
Proof. Assume to the contrary that $n=3 p$ for some odd prime $p$. Without loss of generality we may take the first row of $A$ to be $\left[\begin{array}{llll}1 & 1 & \cdots & 1\end{array}\right]$. Since, $A$ is row-orthogonal and its first row is full, $e_{i}$ is even for $i=2, \ldots, n$. Thus, if $p$ divides $e_{i}$, then $e_{i}=2 p$. For each $j$, set $K_{j}=\left\{i: a_{i j} \neq 0\right.$ and $\left.e_{i}=2 p\right\}$, and $k_{j}=\left|K_{j}\right|$.

By Proposition 3, we have

$$
\frac{1}{3 p}+\frac{k_{j}}{2 p}+\sum_{i \notin\left(K_{j} \cup\{1\}\right)} \frac{1}{e_{i}}=1 .
$$

By Proposition 4

$$
\frac{1}{3 p}+\frac{k_{j}}{2 p} \in Q_{p}
$$

It follows that

$$
2+3 k_{j} \equiv 0 \bmod p .
$$

Let $\ell$ be the unique integer with $0 \leq \ell \leq p-1$ and $2+3 \ell \equiv 0 \bmod p$. Since $p \geq 3, \ell \neq 0$. We have $k_{j} \equiv \ell \bmod p$, and since (1) implies that $k_{j}<2 p$, and since $k_{j}$ is nonnegative, either $k_{j}=\ell$ or $k_{j}=\ell+p$. Since $1 \leq \ell \leq p-1$ and $p \mid(3 \ell+2)$, we have

$$
3 \ell+2=p \text { or } 3 \ell+2=2 p .
$$

First suppose there is a $j$ with $k_{j}=\ell$. Without loss of generality, we may assume that $j=1$, the first $j+1$ entries of column one are +1 , and $e_{2}=\cdots=e_{\ell+1}=2 p$.

For subsets $\alpha$ and $\beta$ of $\{1,2, \ldots, n\}$ the submatrix of $Q$ whose row indices belong to $\alpha$ and whose column indices belong to $\beta$ is denoted by $Q[\alpha, \beta]$. For $m \neq i$, let $a_{m}$, respectively $b_{m}$, denote the number of times $\left[\begin{array}{ll}\frac{1}{\sqrt{2 p}} & \frac{1}{\sqrt{2 p}}\end{array}\right]$, respectively $\left[\begin{array}{ll}\frac{1}{\sqrt{2 p}} & -\frac{1}{\sqrt{2 p}}\end{array}\right]$, occurs as a row of $Q[\{1, \ldots, n\},\{1, m\}]$. Since the columns of $Q$ are mutually orthogonal and of length 1, Propositions 3 and 4 imply that

$$
2+3\left(a_{m}-b_{m}\right) \equiv 0 \bmod p,
$$

and thus

$$
a_{m}-b_{m} \equiv \ell \bmod p
$$

As

$$
\left|a_{m}-b_{m}\right| \leq \ell<p
$$

we conclude that either

$$
a_{m}-b_{m}=\ell \text { or } a_{m}-b_{m}=\ell-p .
$$

Let $c_{2}, \ldots, c_{s}$ be the columns of $A$ with $a_{m}-b_{m}=\ell$. Note that if $a_{m}-b_{m}=\ell$ then, $a_{m}=$ $\ell$ and $b_{m}=0$, since $a_{m}$ and $b_{m}$ are nonnegative and $a_{m}+b_{m} \leq \ell$. Set $\beta=\left\{1, c_{2}, \ldots, c_{s}\right\}$, $X=A[\{2, \ldots, \ell+1\}, \beta], Y=A[\{2, \ldots, \ell\}, \bar{\beta}]$, and $Z=A[\{2, \ldots, \ell\},\{1,2, \ldots, n\}]$.

There are $s \ell$ 1's in $X$. Let $y_{+}$, respectively $y_{-}$, denote the number of 1 's, respectively -1 's, in $Y$. Each row of $Z$ has $2 p$ nonzero entries, and is orthogonal to the vector of all 1 's. Thus each row in $Z$ contains $p 1$ 's and $p-1$ 's, there are $\ell p 1$ 's and $\ell p-1$ 's in $Z$, and

$$
s \ell+y_{+}=\ell p=y_{-} .
$$


Thus,

$$
s \ell=y_{-}-y_{+} \text {and } s \leq p .
$$

Since each column of $Y$ has sum $\ell-p$,

$$
y_{+}-y_{-}=(\ell-p)(n-s) .
$$

Thus

$$
(n-s) \ell=(n-s) p+y_{+}-y_{-} .
$$

Thus by (3) and (4),

$$
3 p \ell=n \ell=s \ell+(n-s) \ell=(n-s) p .
$$

This implies that $s=3 p-3 \ell$. Equation (2) implies $s \geq 3 p-(2 p-2)=p+2$, which contradicts (3). Therefore every column of $Q$ has $\ell+p$ entries equal to $\frac{1}{\sqrt{2 p}}$.

Let $\gamma=\{i$ : row $i$ of $A$ has $2 p$ nonzero entries $\}$. Then $A[\gamma,\{1,2, \ldots, n\}]$ has $2 p$ nonzero entries in each row, and $\ell+p$ nonzero entries in each column. Thus $3 p(\ell+p)=$ $2 p|\gamma|$. Since $p$ is odd, $\ell$ must be odd. In particular, $3 \ell+2 \neq 2 p$. From (2) we conclude that $\ell=\frac{p-2}{3}$.

Let $a_{j}$ and $b_{j}$ be as previously defined. Then by Propositions 3 and 4 , we see that for $j \neq 1$

$$
a_{j}-b_{j} \equiv \ell \bmod p .
$$

As $\left|a_{j}-b_{j}\right| \leq \ell+p=(4 p-2) / 3$, we have

$$
a_{j}-b_{j} \in\left\{\frac{4 p-2}{3}, \frac{p-2}{3}, \frac{-2 p-2}{3}\right\} .
$$

For each $j$, let $x^{j}=A[\{i: i \notin \gamma \cup\{1\}\} ;\{j\}]$.

First suppose that there exists a column $j \neq 1$ with $a_{j}-b_{j}=(4 p-2) / 3=\ell+p$. Then by Proposition 3, we have

$$
\frac{1}{3 p}+\frac{\ell+p}{2 p}+x^{1} \cdot x^{j}=0 .
$$

This implies that

$$
\left|x^{1} \cdot x^{j}\right|=\frac{2+3 \ell+3 p}{6 p}=\frac{2}{3} .
$$

Similarly,

$$
\left\|x^{1}\right\|^{2}=\left\|x^{j}\right\|^{2}=\frac{3 p-2-3 \ell}{6 p}=\frac{1}{3} .
$$

By the Cauchy-Schwartz inequality, $2 / 3=\left|x^{1} \cdot x^{j}\right| \leq\left\|x^{1}\right\|\left\|x^{j}\right\|=1 / 3$, which is a contradiction. Therefore, for each $j \neq 1$, either $a_{j}-b_{j}=(-2 p-2) / 3=\ell-p$ or $a_{j}-b_{j}=(p-2) / 3=\ell$.

Let $s$ be the number of $j$ such that $a_{j}-b_{j}=\ell$. The matrix $A\left[K_{1},\{1,2, \ldots, n\}\right]$ has row sums 0,1 column with sum $\ell+p, s$ columns with sum $\ell$, and $3 p-1-s$ columns with 
sum $\ell-p$. Hence,

$$
\begin{aligned}
0 & =(\ell+p)+s \ell+(n-1-s)(\ell-p) \\
& =2 p+n \ell-n p+s p \\
& =p(s-3 p+3 \ell+2)
\end{aligned}
$$

which implies that $s=2 p$.

Since the rows of $A$ are mutually orthogonal,

$$
0=\sum_{i_{1}, i_{2} \in \gamma, i_{1}<i_{2}} A\left[\left\{i_{1}\right\},\{1,2 \ldots, n\}\right] \cdot A\left[\left\{i_{2}\right\},\{1,2 \ldots, n\}\right] .
$$

Column 1 of $A$ contributes $\left(\begin{array}{c}\ell+p \\ 2\end{array}\right)$ and column $j(j \neq 1)$ of $A$ contributes $\left(\begin{array}{c}a_{j} \\ 2\end{array}\right)+\left(\begin{array}{c}b_{j} \\ 2\end{array}\right)-a_{j} b_{j}$ to the sum on the righthand-side of (5). Hence,

$$
0=\left(\begin{array}{c}
\ell+p \\
2
\end{array}\right)+\sum_{j=2}^{n}\left(\left(\begin{array}{c}
a_{j} \\
2
\end{array}\right)+\left(\begin{array}{c}
b_{j} \\
2
\end{array}\right)-a_{j} b_{j}\right) .
$$

Note that $a_{j}+b_{j} \leq p+\ell=(4 p-2) / 3$ and hence

$$
\begin{aligned}
\left(\begin{array}{c}
a_{j} \\
2
\end{array}\right)+\left(\begin{array}{c}
b_{j} \\
2
\end{array}\right)-a_{j} b_{j} & =\frac{1}{2}\left(\left(a_{j}-b_{j}\right)^{2}-\left(a_{j}+b_{j}\right)\right) \\
& \geq \begin{cases}\frac{1}{2}\left[\left(\frac{p-2}{3}\right)^{2}-\frac{4 p-2}{3}\right] & \text { if } a_{j}-b_{j}=\frac{p-2}{3} \\
\frac{1}{2}\left[\left(\frac{2 p+2}{3}\right)^{2}-\frac{4 p-2}{3}\right] & \text { if } a_{j}-b_{j}=\frac{-2 p-2}{3}\end{cases}
\end{aligned}
$$

Hence, by (6) and the fact $s=2 p$, we have

$$
\begin{aligned}
0 & \geq\left(\begin{array}{c}
\ell+p \\
2
\end{array}\right)+\frac{s}{2}\left(\left(\frac{p-2}{3}\right)^{2}-\frac{4 p-2}{3}\right)+\frac{3 p-1-s}{2}\left(\left(\frac{2 p+2}{3}\right)^{2}-\frac{4 p-2}{3}\right) \\
& =\left(\begin{array}{c}
(4 p-2) / 3 \\
2
\end{array}\right)+p\left(\left(\frac{p-2}{3}\right)^{2}-\frac{4 p-2}{3}\right)+\frac{p-1}{2}\left(\left(\frac{2 p+2}{3}\right)^{2}-\frac{4 p-2}{3}\right) \\
& =\frac{p\left(p^{2}-4 p+1\right)}{3} .
\end{aligned}
$$

It is easy to verify that $p^{2}-4 p+1>0$ for $p \geq 4$. We are led to the contradiction that $p$ is an odd prime with $p<4$ and $\ell=(p-2) / 3$ an integer. Therefore, $n$ is not of the form $3 p$ where $p$ is an odd prime. 


\section{References}

[C] R. Craigen, Weighing Matrices and Conference Matrices in Handbook of Combinatorial Designs (C. Colbourn and J. Dinitz eds), CRC Press, 1996.

[GS] A. V. Geramita and J. Seberry, Orthogonal Designs, Marcel Dekker, New York, 1979.

[GZ] P. M. Gibson and G.H. Zhang, Column orthogonal (0,1,-1)-matrices. Linear and Multilinear Algebra 45 (1999), 291-316.

[HJ] R.A. Horn and C.R. Johnson, Matrix Analysis, Cambridge University Press, Cambridge, 1985.

[HKS] J. Horton, C. Koukouvinos, and J. Seberry, A search for Hadamard matrices constructed from Williamson matrices, Bull. Institute of Combinatorics and its Applications, 35, (2002), 75-88.

[WSW] W. D. Wallis, Anne Penfold Street, and J. Seberry Wallis, Combinatorics: Room Squares, Sum-Free Sets, Hadamard Matrices, Springer-Verlag, Berlin 1972. 